\title{
General anesthesia without neuromuscular blockade for a child with mitochondrial myopathy
}

\author{
Younghoon Jeon ${ }^{1}$, Hyunjae $\mathrm{Kim}^{2}$, and Byungdoo Son ${ }^{2}$ \\ Department of Anesthesiology and Pain Medicine, ${ }^{1}$ School of Dentistry, Kyungpook National University, ${ }^{2}$ School of Medicine, \\ Kyungpook National University, Daegu, Korea
}

Mitochondrial myopathies are rare diseases caused by disturbance of the mitochondrial chain of respiration or oxidative phosphorylation [1]. The mitochondria are a potential site of action for general anesthetic agents, and it is feasible that children with mitochondrial disease will respond abnormally to anesthetic drugs [2]. We experienced a case of successful management of a 2-year-old boy with mitochondrial myopathy under general anesthesia without the use of neuromuscular blockade. A 2-year-old boy weighing $10 \mathrm{~kg}$ and $87 \mathrm{~cm}$ in height, diagnosed with complex I deficiency mitochondrial myopathy by a pediatrician, was admitted for elective cochlear implantation. Neuromuscular examination showed mild muscle weakness and hypotonia, but the patient could control his head and sit alone. He During the preoperative fasting period, an intravenous cannula was inserted and a $500 \mathrm{ml}$ solution containing $3 \%$ dextrose and $38.5 \mathrm{mEq} /$ $\mathrm{L} \mathrm{NaCl}$ was infused at a rate of $40 \mathrm{ml} / \mathrm{h}$ to maintain glucose levels and prevent catabolism. The patient's fasting plasma lactate and glucose levels were $0.9 \mathrm{mmol} / \mathrm{L}$, and $90 \mathrm{mg} / \mathrm{dl}$, respectively. Immediately before entering the operating room, the patient was premedicated with midazolam $0.03 \mathrm{mg} / \mathrm{kg}$ intravenously. Intraoperative routine monitoring included electrocardiography, pulse oximetry, blood pressure, end-tidal capnography and esophageal temperature. Before induction, vital signs showed blood pressure 96/60 $\mathrm{mmHg}$, heart rate 145 beats/min, and oxygen saturation $100 \%$. General anesthesia was induced with 5 vol\% of sevoflurane in $100 \%$ oxygen, and the patient was intubated. Anesthesia was maintained with
$1.5-3$ vol\% of sevoflurane in $66 \%$ nitrous oxide in oxygen. No neuromuscular blocking agent was administered. Intraoperative vital signs kept showed; blood pressure 90/40-100/55 mmHg, heart rate $130-150$ beats/min, end-tidal CO2 38-40 $\mathrm{mmHg}$, body temperature $35.9-36.6^{\circ} \mathrm{C}$, and oxygen saturation $100 \%$. On completion of surgery, sevoflurane was discontinued and the patient was ventilated with $100 \%$ oxygen $5 \mathrm{~L} / \mathrm{min}$ with a manual Ambu bag. After about three minutes the patient started to breathe spontaneously. Six minutes later, tracheal extubation was performed and the patient was transferred to the postanesthetic care unit, where he was monitored with pulse oximetry for 24 hours. Following the operation, the patient's plasma lactate and glucose levels were $1.1 \mathrm{mmol} / \mathrm{L}$, and $95 \mathrm{mg} / \mathrm{dl}$, respectively. There were no adverse events for 24 hours after anesthesia, and the patient was discharged from the hospital the following week.

Mitochondrial myopathies involve the central nervous system, peripheral nervous system, heart, liver, kidneys, endocrine glands, gastrointestinal tract, and bone marrow. Therefore, the symptoms include encephalopathy, seizures, visual disturbances, hepatic and renal failure, and delayed development. Our patient suffered from delayed development, sensorineural deafness, and hypotonia. Although a lactate/ pyruvate ratio greater than 20 is suggestive of respiratory chain problems, a normal lactate/pyruvate ratio is often reported in these patients [1]. In this case, the patient had a low mitochondrial DNA I titer, but a normal lactate/pyruvate ratio. Premedication with midazolam was considered to be of

Corresponding author: Younghoon Jeon, M.D., Department of Anesthesiology and Pain Medicine, School of Dentistry, Kyungpook National University, 188-1, Samduk-dong 2ga, Jung-gu, Daegu 700-721, Korea. Tel: 82-53-420-5863, Fax: 82-53-426-2760, E-mail: jeon68@knu.ac.kr (c) This is an open-access article distributed under the terms of the Creative Commons Attribution Non-Commercial License (http:// creativecommons.org/licenses/by-nc/3.0/), which permits unrestricted non-commercial use, distribution, and reproduction in any medium, provided the original work is properly cited. 
great benefit in this patient, because deafness and cognitive impairment make communication and evaluation difficult, with an increased potential for agitation during the perioperative period [3]. Anesthetic management of patients with mitochondrial myopathies should begin with careful investigation of the medical history and a complete physical examination to exclude possible associated comorbidities, as well as hypotonia, cardiac dysrhythmias, epileptic seizures, stroke-like episodes, gastrointestinal dysmotility, diabetes, and lactic -acidosis [2,3]. Patients with mitochondrial myopathies may be more sensitive to neuromuscular relaxants, so neuromuscular blockade should be closely monitored or even avoided $[2,3]$. The increased sensitivity of these patients to nondepolarizing neuromuscular blockade and inadequate reversal by anticholinesterases can result in delayed recovery. In addition, malignant hyperthermia has been associated with mitochondrial myopathies [4]. In the case described here, the patient showed mild muscle weakness and hypotonia on neuromuscular examination although he could control his head and sit alone. Therefore, we did not use any neuromuscular blockade and selected sevoflurane for induction and maintenance of anesthesia. Although arrhythmias with halothane have been reported in patients with mitochondrial myopathies [5], sevoflurane has been used uneventfully in patients with these diseases [3]. Its low solubility and low pungency allow a rapid, smooth inhalational induction without the need for neuromuscular blocking drugs. In addition, sevoflurane elimination does not depend on renal and hepatic pathways, which could be compromised in these patients.

In conclusion, general anesthesia under sevoflurane without the use of neuromuscular blockade can be safely used for anesthetic management of patients with mitochondrial myopathy.

\section{References}

1. Tsao CY, Mendell JR, Lo WD, Luquette M, Rusin J. Mitochondrial respiratory-chain defects presenting as nonspecific features in children. J Child Neurol 2000; 15: 445-8.

2. Shipton EA, Prosser DO. Mitochondrial myopathies and anaesthesia. Eur J Anaesthesiol 2004; 21: 173-8.

3. Vilela H, Garcia-Fernandez J, Parodi E, Reinoso-Barbero F, Durán P, Gilsanz F. Anesthetic management of a patient with MERRF syndrome. Paediatr Anaesth 2005; 15: 77-9.

4. Allison KR. Muscular dystrophy versus mitochondrial myopathy: the dilemma of the undiagnosed hypotonic child. Paediatr Anaesth 2007; 17: 1-6.

5. Estes R, Ginsberg B, Block SEC. Anaesthesia and the Kearns-Sayre syndrome. Paediatr Anaesth 1993; 33: 307-11. 